The maximum value of $m$ is $l^{2}-l+1$, $^{*}$ and the largest value of $l$ is the largest integer in $\frac{1}{2} n$. When $n$ is even, say $2 l$, these curves are already included among those treated before for $r=\frac{1}{2} n-1$. By taking smaller values for $l$, curves of all the higher genera can be constructed.

3 . When $n$ is odd, say $n=2 l+1$, the lowest genus obtainable directly is $l^{2}-1$, while the highest one from the space curve is $l^{2}-l$. By passing a pencil of $c_{t+1}$ through the $l^{2}$ base points of a pencil of $c$, the lower genus can be obtained, and the higher one by projection. To obtain the intermediate cases, pass two $c_{l}$ through $l-1$ points on a fixed straight line $c_{1}$. Through $\kappa$ of the $l^{2}-l+1$ remaining points of intersection, the same $l-1$ points on $c_{1}$, and two other points on $c_{1}$ pass a pencil of $c_{l+2}$. The two pencils are now to be made projective in such a way that corresponding curves intersect on $c_{1}$. The locus of the point of intersection will be a $c_{21+2}$, but $c_{1}$ will be a factor. The remaining $c_{2 l+1}$ will have $\kappa+l-1$ double points, and no other singularities. $\kappa$ can have any value from 0 to $l^{2}-l+1$. This completes the solution of the problem. $\dagger$

\title{
CoRnelL UNIVERSTTY,
}

July, 1908.

\section{ON PERIODIC LINEAR SUBSTITU'TIONS WHOSE COEFFICIENTS ARE INTEGERS.}

BY DR. ARTHUR RANUM.

(Read before the American Mathematical Society, September 6, 1907.)

1. THE object of this note is to call attention to one or two theorems that follow easily from the results of my paper in this Bulletis, April, 1907, volume 13, pages 336-345, by taking into account a theorem of Minkowski's given in Crelle,

* C. Kupper : "Ueber das Vorkommen von linearen Schaaren..." Sitzungsbericht der Böhm. Gesell., Prag, 1892, pp. 264-262. In my article "On curves having a net of minimum adjoint curves," BuLcETIN, vol. 14, page 70 (1907) I showed how such a net of curves can be actually constructed by rational operations.

† Since the basis points are not independent, Cayley's theorems regarding the configuration of residual points of intersection do not apply. See Küpper,

"Bestimmung der Maximalbasis $B$ für eine irreduoible $\mu$-fache Mannig. faltigkeit von Curven $n$ ter Ordnung," Monatshefte für Math. u. Physik, vol. 6, (1895), pp. 5-11, and my own paper, "On birational transformations of curves of high genus," Amer. Jour. Math. vol. 30 (1908), pp. 10-20. 
volume 100, pages 449-456, and volume 101, pages 196-202. They relate to linear homogeneous substitutions of finite period in $n$ variables, whose coefficients are integers, and whose determinants are different from zero and are therefore \pm 1 .

2. Two such linear substitutions (matrices) are said to be congruent $\bmod k$, if their corresponding coefficients (elements) are congruent $\bmod k$. Letting $I$ stand for the identical matrix, Minkowski's theorem may be stated as follows :

The only periodic matrix that is congruent to $I \bmod k$, if $k>2$, is I itself, of period 1; while the finite periods of the matrices that are congruent to $I \bmod \mathscr{Z}$, are 1 and $\mathscr{\mathscr { Z }}$.

As illustrations of this theorem, consider the matrix $\left(\begin{array}{cc}1+3 a & 3 b \\ 3 c & 1+3 d\end{array}\right)$, which is congruent to $\left(\begin{array}{ll}1 & 0 \\ 0 & 1\end{array}\right) \bmod 3$, but is of infinite period unless $a=b=c=d=0$; and on the other hand the matrix $\left(\begin{array}{ll}1 & 0 \\ 2 c & -1\end{array}\right)$, which is congruent to $\left(\begin{array}{ll}1 & 0 \\ 0 & 1\end{array}\right) \bmod 2$, and is of period 2.

3. Let us now determine what happens to the period of a matrix when it is considered as belonging to a linear congruence group, or Jordan group, whose modulus is $k$. In that case any two matrices which are congruent mod $k$ become identical, and a matrix that is congruent to $I \bmod k$ becomes $I$ itself. The period of every matrix becomes finite, whether it was originally finite or infinite; if it was originally finite $(=m)$, it not only remains finite, but obviously becomes a divisor of $m$. The following theorem can now be readily proved:

If a matrix $L$ whose elements are integers is of finite period $m$, then the corresponding matrix $L^{\prime}$, belonging to the Jordan group whose modulus is $k$, is also of period $m$, if $k>\mathscr{2}$; while if $k=\mathscr{2}$, its period is either $m$ or $\frac{1}{2} m$.

Proof. Let the period of $L^{\prime}$ be $m^{\prime}$, and put $m / m^{\prime}=r$, which is an integer. Then $\left(L^{\prime}\right)^{m^{\prime}}=I$, and $I^{m^{\prime}}$, which is clearly of period $r$, is at the same time congruent to $I \bmod k$. Therefore, by Minkowski's theorem, we see that if $k>2, r=1$ and $m^{\prime}=m$; while if $k=2, r=1$ or 2 , and $m^{\prime}=m$ or $\frac{1}{2} m$.

As an illustration of this theorem, consider the matrix $\left(\begin{array}{c}0-1 \\ 1\end{array}\right)$, whose period, irrespective of any modulus, is 6 ; with respect to the modulus 3 its period remains 6 , while with respect to the modulus 2 its period becomes 3 .

4. This result furnishes a short cut to the following theorem, which was otherwise proved in my paper in the Transactions, April, 1908, volume 9, page 191, Corollary 2.

The highest prime period of an n-ary matrix whose elements are integers cannot be greater than $n+1$. 
Proof. Let $L$ be an $n$-ary matrix of period $p$, a prime, and suppose that $L^{\prime}$ is the corresponding matrix of the Jordan group whose modulus is $p^{a}(a>1)$; by the theorem of $\S 3, L^{\prime}$ is also of period $p$. Now suppose that $p$ could be $>n+1$ (and therefore $>2$ ); then, in view of theorems 2 and 5 of the BULLETIN paper referred to above (pages 339 and 342, respectively),* the matrix $L_{1}^{\prime}$, being excluded from $H-H_{1}$ and $H_{1}-H_{2}$ (in the notation of that paper), would necessarily belong to $H_{a-1}-I$, and would be congruent to $I \bmod p^{a-1}$. Therefore $L$ would itself be congruent to $I \bmod p^{a-1}$. But by Minkowski's theorem this is impossible.

5. In a similar manner, by reference to theorems 3 and 4 of the same Bulletin paper, it is easily seen that if $n \leqq p^{a}$, where $p$ is an odd prime, then the period of an $n$-ary matrix whose elements are integers cannot be a power of $p$ higher than the $a$ th. $†$

6 . The general problem of finding all the finite periods of $n$-ary matrices whose elements are integers can be solved at once by observing that they are identically the same as the finite periods of $n$-ary matrices whose elements are rational numbers. In other words, the use of rational fractions as the elements of a matrix does not make possible any finite periods not already existing in the case of matrices whose elements are integers.

This follows from the fact (Transactions, 1. c., §\$21-27) that for every matrix of finite period with rational elements there exists a canonical form, of the same period, whose elements are integers.

Accordingly, the results given in $\S \S 10-20$ of the paper just referred to furnish a complete solution of the problem of this section.

Cornell University, June, 1908.

* Minkowski's theorem itself can also be derived from the results given in that paper.

t It is to be noticed, however, that this result is not so strong or complete as the corresponding one given in the Transactions, l. c., p. 191, Corollary 2. 